included. SSc patients fulfilled ACR/EULAR 2013 criteria. Anthropometric parameters and body composition were assessed (by densitometry: iDXA Lunar, and by bioelectric impedance: BIA-2000-M), and physical activity was evaluated using Human Activity Profile (HAP) questionnaire. Routine biochemistry analysis was performed after 8 hours of fasting. Pulmonary function and diffusing capacity of lung were examined, disease activity was evaluated by ESSG activity index. Data are presented as mean $\pm S D$.

Results: Compared to $\mathrm{HC}$, patients with SSc had significantly lower body-mass index (BMl:27.9 \pm 8.3 vs. $22.4 \pm 4.3 \mathrm{~kg} / \mathrm{m}^{2}, \mathrm{p}<0.0001$ ) and body fat $\%$ assessed by both iDXA (BF\%:38.0 \pm 7.6 vs. $32.6 \% \pm 8.2 \%, \mathrm{p}<0.0001)$ and $\mathrm{BIA}(\mathrm{BF} \%: 31.3 \pm 7.6$ vs. $24.3 \% \pm 7.9 \%, p<0.0001)$, and decreased visceral fat weight $(1.0 \pm 0.8$ vs. 0.5 $\pm 0.5 \mathrm{~kg}, \mathrm{p}=0.001$ ). Compared to HC, SSc patients also demonstrated significantly decreased lean body mass assessed by both iDXA (LBM:51.9 \pm 8.4 vs. 47.8 $\pm 7.0 \mathrm{~kg}, \mathrm{p}=0.005$ ) and $\mathrm{BIA}$ (LBM:45.4 \pm 7.3 vs. $40.9 \pm 6.8 \mathrm{~kg}, \mathrm{p}=0.005)$, significantly lower bone mineral density (BMD:1.2 \pm 0.1 vs. $1.0 \pm 0.1 \mathrm{~g} / \mathrm{cm}^{2}, \mathrm{p}<0.0001$ ), and had increased extracellular mass/body cell mass (ECM/BCM) ratio ( $1.03 \pm 0.1$ vs. 1.28 $\pm 0.4, p<0.0001$ ), which reflects deteriorated nutritional status and worse muscle predispositions for physical exercise, aerobic fitness/performance. Increased ECM/BCM in SSc patients positively correlated with disease activity (ESSG: $r=0.273, p=0.0439$ ), skin score (mRSS: $r=0.371, p=0.0045$ ) and inflammation (CRP: $r=0.292, p=0.0278$; ESR: $r=0.302, p=0.0226$ ). Increased $E C M / B C M$ was also associated with worse quality of life (HAQ:r=0.438, $p=0.0007$; SHAQ: $r=0.268, p=0.0436$ ), fatigue (FSS: $r=0.366, p=0.0040$ ), and worse ability to perform physical activity (HAP: $r=-0.644, \mathrm{p}<0.0001)$. Disease activity (ESSG) negatively correlated with BF\% by iDXA ( $r=-0.324, p=0.0138)$. Physical activity (HAP) positively correlated with BMD $(r=0.280, p=0.032)$. There was no significant correlation of lung involvement with alterations of body composition.

Conclusions: Compared to healthy age-/sex-matched individuals we found significant negative changes in body composition of our SSc patients, which are associated with their disease activity and physical activity, and could reflect their nutritional status, and gastrointestinal and musculoskeletal involvement. We found no significant association between lung involvement and changes of body composition.

Acknowledgements: Supported by AZV-16-33574A and SVV-260373

Disclosure of Interest: None declared

DOI: 10.1136/annrheumdis-2018-eular.6978

\begin{tabular}{|l|l}
\hline AB0800 & CHANGES OF BODY COMPOSITION IN MYOSITIS \\
& PATIENTS ARE ASSOCIATED WITH DISEASE \\
& DURATION, INFLAMMATORY STATUS, SKELETAL \\
& MUSCLE INVOLVEMENT AND PHYSICAL ACTIVITY
\end{tabular}

S. Oreska ${ }^{1}$, M. Spiritovic ${ }^{1,2}$, P. Cesak $^{2}$, O. Marecek ${ }^{2}$, H. Storkanova ${ }^{1}$, H. Smucrova ${ }^{1}$, B. Hermankova ${ }^{1,2}$, K. Kubinova ${ }^{1}$, M. Klein ${ }^{1}$, L. Vernerova ${ }^{1}$, O. Ruzikova ${ }^{1}$, R. Becvar ${ }^{1}$, K. Pavelka ${ }^{1}$, L. Senolt ${ }^{1}$, H. Mann ${ }^{1}$, J. Vencovsky ${ }^{1}$, M. Tomcik'. ' ${ }^{1}$ Department of Rheumatology, 1st Medical Faculty, Charles University, Institute of Rheumatology; ${ }^{2}$ Department of Physiotherapy, Faculty of Physical Education and Sport, Charles University, Prague, Czech Republic

Background: Idiopathic inflammatory myopathies (IIM) are characterised by inflammation and atrophy of skeletal muscles, pulmonary and articular involvement, which limit the mobility/self-sufficiency of patients, and can have a negative impact on body composition.

Objectives: To assess body composition and physical activity of IIM patients and healthy controls $(\mathrm{HC})$

Methods: 54 patients with IIM (45 females/9 males; mean age 57.7; disease duration 5.8 years; polymyositis (PM, 22)/dermatomyositis (DM, 25)/necrotizing myopathy (IMNM, 7)) and 54 age-/sex-matched HC (45 females/9 males, mean age 57.7) without rheumatic diseases were included. PM/DM patients fulfilled Bohan/Peter criteria for PM/DM. Anthropometric parameters and body composition were assessed (by densitometry: iDXA Lunar, and by bioelectric impedance: BIA2000-M), and physical activity was evaluated using Human Activity Profile (HAP) questionnaire. Routine biochemistry analysis was performed after 8 hours of fasting. Muscle involvement was evaluated by manual muscle test (MMT-8) and functional index $2(\mathrm{FI} 2)$. Data are presented as mean $\pm \mathrm{SD}$.

Results: Compared to $\mathrm{HC}$, patients with IIM had a trend towards significantly increased body fat $\%$ as assessed by iDXA (BF\%: $39.9 \pm 7.1$ vs. $42.4 \% \pm 7.1 \%$, $\mathrm{p}=0.077$ ), but significantly decreased lean body mass as assessed both by iDXA (LBM: $45.6 \pm 8.1$ vs. $40.6 \pm 7.2 \mathrm{~kg}, \mathrm{p}=0.001$ ) and BIA (LBM: $52.6 \pm 8.8$ vs. 48.7 $\pm 9.0 \mathrm{~kg}, \mathrm{p}=0.023$ ), and increased extracellular mass/body cell mass (ECM/BCM) ratio ( $1.06 \pm 0.15$ vs. $1.44 \pm 0.42, p<0.0001)$, which reflects worse muscle predispositions for physical exercise, aerobic fitness/performance, and also deteriorating nutritional status. Compared to $\mathrm{HC}$, IIM patients had significantly lower bone mineral density (BMD: $1.2 \pm 0.1$ vs. $1.1 \pm 0.1 \mathrm{~g} / \mathrm{cm}^{2}, p<0.001$ ). Disease duration negatively correlated with BMD $(r=-0.392, p=0.004)$ and LBM-BIA $(r=-0.272$, $p=0.047)$. CRP was positively associated with $B F \%$ assessed both by DEXA $(r=0.276, p=0.035)$ and BIA $(r=0.306, p=0.025)$. Higher $B F \%>D E X A$ was associated with worse physical endurance $(F \mid 2: r=-0.311, p=0.026)$ and worse ability to perform physical activity (HAP: $r=-0.292, p=0.032$ ). MMT-8 score negatively correlated with $\mathrm{ECM} / \mathrm{BCM}$ ratio $(\mathrm{r}=-0.385, \mathrm{p}=0.006)$.

Conclusions: Compared to healthy age-/sex-matched individuals we found significant negative changes in body composition of our IIM patients, which are associated with their disease duration, inflammatory status, skeletal muscle involvement, and physical activity, and could reflect their impaired nutritional status and predispositions for physical exercise, aerobic fitness and performance.

Acknowledgements: Supported by AZV-16-33574A and SVV-260373.

Disclosure of Interest: None declared

DOI: 10.1136/annrheumdis-2018-eular.7034

\section{AB0801 SARCOPENIA AND MICROCIRCULATION IN SYSTEMIC SCLEROSIS PATIENTS: A PILOT ASSAY}

S. Paolino, V. Tomatis, A. Casabella, C. Pizzorni, A. Sulli, M. Ghio, B. Ruaro, E. Alessandri, M. Pendolino, C. Cosso, G. Ferrari, S. De Gregorio, M. Cutolo. Department of Internal Medicine, Research Laboratory and Academic Division of Clinical Rheumatology, Department of Internal Medicine, University of Genova, Genoa, Italy, Genoa, Italy

Background: Systemic sclerosis (SSc) patients may present muscle involvement in the form of myositis or non-inflammatory myopathy with different degree of weakness and muscle atrophy. ${ }^{1}$ Sarcopenia is described as a multifactorial syndrome with muscle mass loss associated to functional impairment; according to the anthropometric equation by Baumgartner et al. sarcopenia was defined as relative skeletal muscle mass index $(\mathrm{RSMl})<5.5 \mathrm{Kg} / \mathrm{m}^{2}$ for women and $<7.26 \mathrm{Kg}$ $\mathrm{m}^{2}$ for men. The relationships between sarcopenia and other clinical aspects in SSc are poor investigated.

Objectives: The aim of the study was to evaluate the associations between sarcopenia and other clinical factors in SSc patients.

Methods: 20 female patients fulfilling the ACR 2013 criteria for SSc (mean age $61.7 \pm 13.6$ years, disease duration $86.2 \pm 67.1$ months) were enrolled. The RSM $\left(\mathrm{Kg} / \mathrm{m}^{2}\right)$ was evaluated by dual-energy X-ray absorptiometry scan (Lunar Prodigy). Nail fold videocapillaroscopic (NVC) patterns (early, active, late) were analysed. Serum $25(\mathrm{OH})_{2} \mathrm{D}$ concentration was tested by immunofluorescence. Non parametric statistical tests were used.

Results: Patients showed a modified Rodnan skin score (mRSS) 12.2 \pm 7.7 RSMI $6.01 \pm 0.97 \mathrm{Kg} / \mathrm{m}^{2}$, serum vitamin $\mathrm{D}\left(25(\mathrm{OH})_{2} \mathrm{D}\right) 22.01 \pm 13.1 \mathrm{ng} / \mathrm{dL}$ and $\mathrm{CPK}$ $70.16 \pm 31.8 \mathrm{U} / \mathrm{L}$. In this cohort $23 \%$ of SSc patients were found affected by sarcopenia, and almost $42 \%$ showed the most advanced level of microvascular damage, as characterised by the NVC late pattern. However, no statistically relevant correlations was observed between RSMI, BMI, age, disease duration, CPK, mRSS, $25(\mathrm{OH})_{2} \mathrm{D}$ and active or late NVC patterns. Comparing age, disease dura tion, CPK and mRSS in both sarcopenic and non sarcopenic SSc patients there was no difference between the groups, however sarcopenic patients showed statistically significant lower BMI $(p=0.02)$, lower RSMI $(p=0.0008$ and higher 25 $(\mathrm{OH})_{2} \mathrm{D}$ serum concentrations $(p=0.01)$. Particularly, RSMI showed a strong negative correlation with age $(p=0.01)$. No statistical differences were found when grouping the patients according to the positivity for serum anti-topoisomerase I $A b$ or according to cutaneous diffuse (dcSSc) or limited (IcSSc) systemic sclerosis.

Conclusions: This pilot study suggests that sarcopenia seems present in almost $25 \%$ of the SSc patients, particularly in a condition of advanced microcirculation failure (late NVC pattern), however does not seem to correlate with skin fibrosis or disease duration. The study had some limitations due to absence of control group and to the small patient sample analysed. Further larger studies would be necessary to better investigate the role of sarcopenia in SSc.

\section{REFERENCE:}

[1] Ranque, B., Authier, F.J., Berezne, A., Guillevin, L., Mouthon, L., 2007. Ann. N. Y. Acad. Sci. 1108, 268-282

Disclosure of Interest: None declared

DOI: 10.1136/annrheumdis-2018-eular.6658

\section{AB0802 OSTEOPOROSIS IN SYSTEMIC SCLEROSIS: CASE- CONTROL STUDY WITH A FRENCH OFELY COHORT} AND RISK FACTORS

S. Thietart ${ }^{1}$, M. Gatfosse ${ }^{1}$, E. Sornay-Rendu ${ }^{2}$, L. Lemeunier ${ }^{3}$, P. Delmaire ${ }^{4}$ J. Sellam ${ }^{3}$, F. Berenbaum ${ }^{3}$, O. Fain ${ }^{1}$, R. Chapurlat ${ }^{2}$, A. Mekinian' ${ }^{1}$. ${ }^{1}$ Internal medecine, Hôpital Saint Antoine, AP-HP, Université Paris 6, 75012 Paris, France., Paris; ${ }^{2}$ Unité Inserm UMR 1033, Université de Lyon, Lyon; ${ }^{3}$ Rheumatology; ${ }^{4}$ Nuclear medecine, Hôpital Saint Antoine, AP-HP, Université Paris 6, 75012 Paris, France., Paris, France

Background: Systemic sclerosis (SSc) is a rare autoimmune disorder characterised by a vascular and fibrosing involvement of the skin and internal organs. Data 
about the association between systemic sclerosis (SSc) and osteoporosis (OP) are controversial and scarce about the risk factors of OP in SSc. ${ }^{1,2}$

Objectives: The aim of the study was to determine the OP frequency in SSc and assess its risk factors.

Methods: In a prospective cohort of SSc patients, usual risk factors of OP were assessed, as well as SSc organ involvements: pulmonary, cardiac, skin and renal involvements and SSc treatments. All patients underwent dual energy X-ray absorptiometry: bone mineral density (BMD) was measured at the lumbar spine (LS), femoral neck (FN) and total hip (TH). Osteoporosis was defined as having a T-score inferior to -2.5

Results: Forty-eight patients were included with a median age of 60 years, ${ }^{27-81}$ 41 women $(85.4 \%)$, with a diffuse cutaneous subtype in 13 cases $(27.1 \%)$ and illness duration of 12.6 years $(0.3-41.1)$. Average BMD was $0.98 \pm 0.21$ in LS, 0.84 \pm 0.13 in $\mathrm{FN}$ and $0.86 \pm 0.15$ in TH. OP was found in 19 patients (40\%). Among patients with OP, an associated auto-immune disorder was found in 13 patients $(68.4 \%)$ versus $10(34.5 \%)$ in the non-OP group $(\mathrm{p}=0.04)$, digestive sub-occlusion in 4 patients $(21 \%$ versus 0 patients, $p=0.02)$, and chronic liver disease in 6 patients $(31.6 \%)$ versus $2(6.9 \%, \mathrm{p}=0.04)$. Respiratory explorations found a DLCO of $12.7(6.8-17.2)$ versus $15.6(9.6-32.2)(p=0.007)$, a CVF of $2.47(1.1-4)$ versus $3(1.7-5.9) \quad(p=0.03)$. DAS28-CRP was significantly higher $(2.29(1.53-4.46)$ in the OP group versus $1.86(0-3.8)$ in the non-OP group, $p=0.02)$; with a higher frequency of hand X-ray erosions (6 OP patients $(31.6 \%$ ) versus $2(6.9 \%), \mathrm{p}=0.04)$. No difference was found in Rodnan score, SSc subtype, illness duration, malnutrition, treatments (proton pump inhibitors, cyclophosphamide, steroids), autoantibodies profile; nor with the usual OP risk factors: age, smoking, personal or family history of OP fractures, alcohol consumption, weight, BMI, long term steroid treatment, early menopause and thyroid disease. No difference in serum vitamin D, calcium, thyroid hormones and C-reactive protein levels was found between OP and non-OP groups.

Conclusions: Osteoporosis was associated with SSc-related factors such as: articular, digestive and respiratory involvements, and associated auto-immune diseases. Usual OP risk factors where not significantly different between osteoporotic SSc patients and non-osteoporotic patients.

\section{REFERENCES:}

[1] Caimmi C, Caramaschi P, Barausse G, Orsolini G, Idolazzi L, Gatti D, et al. Bone Metabolism in a Large Cohort of Patients with Systemic Sclerosis. Calcif Tissue Int. 2016 Jul 1:99(1):23-9.

[2] Omair MA, Pagnoux C, McDonald-Blumer H, Johnson SR. Low bone density in systemic sclerosis. A systematic review. J Rheumatol. 2013 Nov;40 (11):1881-90.

Disclosure of Interest: None declared DOI: 10.1136/annrheumdis-2018-eular.6167

\section{AB0803 A REAL LIFE EXPERIENCE ON THE EFFICACY AND SAFETY OF MYCOPHENOLATE MOFETIL IN CONNECTIVE TISSUE DISORDER ASSOCIATED INTERSTITIAL LUNG DISEASE - A RETROSPECTIVE STUDY}

S. Santhanam ${ }^{1}$, V. Rahulan ${ }^{2} .{ }^{1}$ Rheumatology; ${ }^{2}$ Pulmonology, Gleneagles global hospital, chennai, India

Background: Interstitial lung disease(ILD) is one of the common extra articular manifestations of various connective tissue disorders(CTD). We don't have enough evidence on the drugs used, except for ILD in systemic sclerosis and the results of the same has been extrapolated to other diseases. Mycophenolate mofetil(MMF) has antiproliferative and anti fibrotic action in addition to anti inflammatory property and hence we wanted to study the efficacy and safety of MMF in our diverse cohort of CTD-ILD.

Objectives: To study the efficacy and safety of mycophenolate mofetil (MMF) in a diverse cohort of patients with connective tissue disease(CTD) associated interstitial lung disease(ILD).
Methods: This is a retrospective observational study with records of outpatients

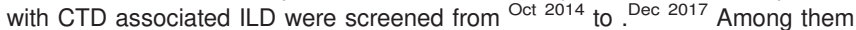
patients with imaging (HRCT chest) documented ILD were included. All patients underwent detailed clinical assessment, serological investigations (baseline blood test, erythrocyte sedimentation rate, $\mathrm{C}$ - reactive protein, rheumatoid factor ACPA Ab, antinuclear antibody, ANA profile, complements), urine routine, pulmonary function test(PFT), HRCT chest and echocardiogram. The response of ILD to treatment(follow up of 2 years) was assessed clinically, radiologically and by PFT.

Results: 54 patients were identified with CTD-ILD of which 33 patients were on MMF. 13 patients were diagnosed with MCTD, 12 with RA, 3 with diffuse cutaneous systemic sclerosis, 2 with SSc/myositis overlap, 1 with primary sjogren's syndrome, 1 with SLE/Sjogren's overlap and 1 with lung dominant CTD(Scl 70+ve). The cohort was divided in to 3 groups - MCTD, RA and others (SSc predominant). Among patients with MCTD, 10 had NSIP pattern of ILD, 3 UIP. In RA, 7 had NSIP and 5 UIP and among others 7 NSIP and 1 UIP. The mean FEV1 and FVC values over 2 years and the treatment response has been discussed in table 1 and Figure 1. FEV1 and FVC had high positive correlations (Pearson correlation, $\mathrm{p}<0.05$ ) with treatment for all groups of diseases. The values go together in the positive direction with treatment. Among the groups, all patients who improved had NSIP pattern and all who worsened had UIP. Though there were numerical differences in the mean values of FEV1 and FVC between two groups (NSIP and UIP), it was not statistically significant (paired ' $t$ ' test, $p>0.05$ ). There was no significant difference in FEV1 and FVC values with treatment between the three groups (one way ANOVA test).
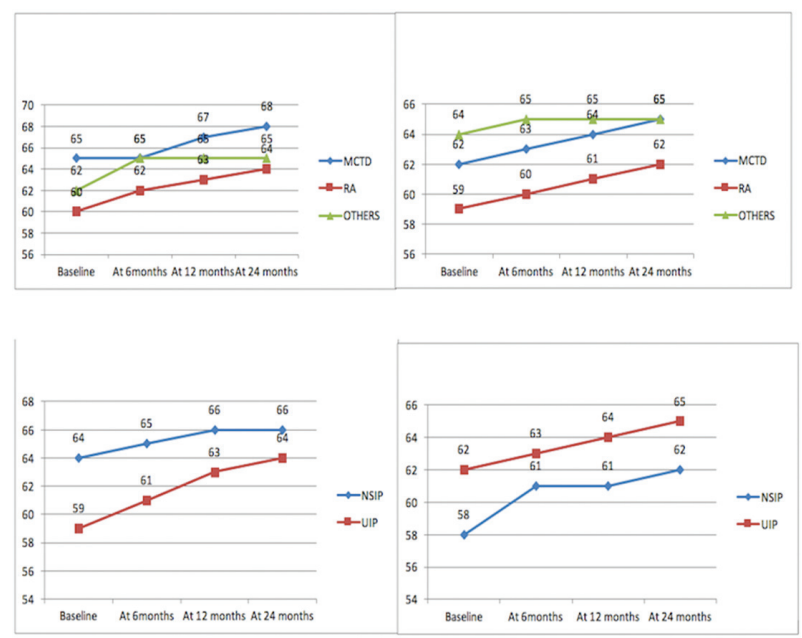

Abstract AB0803 - Figure 1

Conclusions: Treatment with MMF over a median duration of 24 months stabilised the ILD in majority. MMF appears to be efficacious, safe and well tolerated in our diverse cohort of CTD-ILD and needs to be evaluated further in prospective studies with a bigger sample size.

\section{REFERENCE:}

[1] Fischer A, Brown KK, Du Bois RM, et al. Mycophenolate Mofetil Improves Lung Function in Connective Tissue Disease-associated Interstitial Lung Disease. The Journal of rheumatology. 2013;40(5):640-646.

Disclosure of Interest: None declared

DOI: 10.1136/annrheumdis-2018-eular.3395

\begin{tabular}{|c|c|c|c|c|c|c|c|c|c|c|c|}
\hline \multirow[t]{2}{*}{ Variables } & \multicolumn{4}{|c|}{ Mean FEV1 (\%) } & \multicolumn{4}{|c|}{ Mean FVC (\%) } & \multicolumn{3}{|c|}{$\begin{array}{l}\text { ILD after treatment } \\
\text { (PFT and Radiological) }\end{array}$} \\
\hline & Baseline & $\begin{array}{c}\text { At } \\
6 \text { months }\end{array}$ & $\begin{array}{c}\text { At } \\
12 \text { months }\end{array}$ & $\begin{array}{c}\text { At } \\
24 \text { months }\end{array}$ & Baseline & $\begin{array}{c}\text { At } \\
6 \text { months }\end{array}$ & $\begin{array}{c}\text { At } \\
12 \text { months }\end{array}$ & $\begin{array}{c}\text { At } \\
24 \text { months }\end{array}$ & Improved & Stable & Worsened \\
\hline MCTD & 65 & 65 & 67 & 68 & 62 & 63 & 64 & 65 & 4 & 8 & 1 \\
\hline RA & 60 & 62 & 63 & 64 & 59 & 60 & 61 & 62 & 4 & 7 & 1 \\
\hline OTHERS & 62 & 65 & 65 & 65 & 64 & 65 & 65 & 65 & 0 & 7 & 1 \\
\hline
\end{tabular}

